\title{
Targeted Memory Reactivation during Sleep Adaptively Promotes the Strengthening or Weakening of Overlapping Memories
}

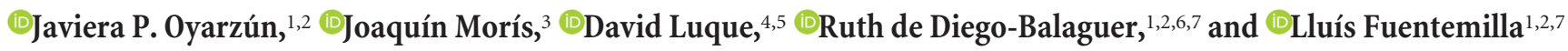 \\ ${ }^{1}$ Department of Cognition, Development and Educational Psychology, University of Barcelona, 08035 Barcelona, Spain, ${ }^{2}$ Cognition and Brain Plasticity \\ Group, IDIBELL, Bellvitge Biomedical Research Institute, L'Hospitalet de Llobregat, 08097 Barcelona, Spain, ${ }^{3}$ Department of Psychology, University of \\ Oviedo, 33003 Oviedo, Spain, ${ }^{4}$ Instituto de Investigación Biomédica de Málaga, University of Málaga, 29071 Málaga, Spain, ${ }^{5}$ School of Psychology, \\ University of New South Wales Sydney, Sydney, New South Wales 2052, Australia, ${ }^{\top}$ CREA, Catalan Institution for Research and Advanced Studies, \\ 08010 Barcelona, Spain, and 'Institute of Neurosciences, University of Barcelona, 08035 Barcelona, Spain
}

System memory consolidation is conceptualized as an active process whereby newly encoded memory representations are strengthened through selective memory reactivation during sleep. However, our learning experience is highly overlapping in content (i.e., shares common elements), and memories of these events are organized in an intricate network of overlapping associated events. It remains to be explored whether and how selective memory reactivation during sleep has an impact on these overlapping memories acquired during awake time. Here, we test in a group of adult women and men the prediction that selective memory reactivation during sleep entails the reactivation of associated events and that this may lead the brain to adaptively regulate whether these associated memories are strengthened or pruned from memory networks on the basis of their relative associative strength with the shared element. Our findings demonstrate the existence of efficient regulatory neural mechanisms governing how complex memory networks are shaped during sleep as a function of their associative memory strength.

Key words: EEG; episodic memory; reactivation; sleep; strengthening; weakening

Significance Statement

Numerous studies have demonstrated that system memory consolidation is an active, selective, and sleep-dependent process in which only subsets of new memories become stabilized through their reactivation. However, the learning experience is highly overlapping in content and thus events are encoded in an intricate network of related memories. It remains to be explored whether and how memory reactivation has an impact on overlapping memories acquired during awake time. Here, we show that sleep memory reactivation promotes strengthening and weakening of overlapping memories based on their associative memory strength. These results suggest the existence of an efficient regulatory neural mechanism that avoids the formation of cluttered memory representation of multiple events and promotes stabilization of complex memory networks.

\section{Introduction}

Memory consolidation is conceptualized as a process triggered by a learning experience whereby newly encoded representations

\footnotetext{
Received Nov. 16, 2016; revised May 2, 2017; accepted May 26, 2017.

Author contributions: J.P.O., J.M., D.L., R.d.D.-B., and L.F. designed research; J.P.O. performed research; J.P.O., J.M., and L.F. analyzed data; J.P.O., J.M., D.L., R.d.D.-B., and L.F. wrote the paper.

This research study was supported by grants from the Spanish Government (PSI2013-46057-P to L.F., PSI201343516-R to J.M.) and the Catalan Government (Generalitat de Catalunya, 2014-SGR-1413). We thank Debbie Talmi and Sid Kouider for their comments on the initial versions of the paper.

The authors declare no competing financial interests.

Correspondence should be addressed to Dr. Javiera Oyarzún. Department of Cognitive, Development and Educational Psychology, University of Barcelona, Feixa Llarga s/n, 08907, L'Hospitalet, Barcelona, Spain. E-mail: javi.oyarzunb@gmail.com.
}

transform into a robust and enduring form (Eichenbaum, 2000). It has been shown that sleep contributes to memory consolidation processes (Maquet, 2001; Diekelmann and Born, 2010; Stickgold and Walker, 2013). One influential hypothesis, supported by studies in rodents (Peyrache et al., 2009; Bendor and Wilson, 2012; Buzsáki, 2015) and human behavioral (Oudiette and Paller, 2013) and neuroimaging data (Peigneux et al., 2004; Bergmann et al., 2012; Deuker et al., 2013), suggests that the core neural mechanism by which memories are consolidated during sleep is their neural reinstatement during off-line periods. In- 
deed, recent findings in rodents have shown that this process engages the interplay of the hippocampus and cortical regions in a cortical-hippocampal-cortical loop of information flow during sleep and that this may influence the identity of memories that are consolidated into long-term (Rothschild et al., 2017). A critical assumption of the systems-level memory reactivation account during sleep is that this process is active (Diekelmann and Born, 2010) and selective (Oudiette et al., 2013), and as such, only a subset of new memories will become strengthened through their reactivation during sleep. However, memories are not isolated in the brain. Because our daily learning experience is highly overlapping in content, memory events are rarely encoded as individual memory traces. Instead, memories that share features are linked in an organized and intricate network of overlapping associated events (Eichenbaum, 2000). Thus, although central to theories of memory consolidation, it remains to be explored whether memory reactivation has an impact on overlapping memories acquired during awake time (Lewis and Durrant, 2011). And if so, what are the governing principles that prevent associative memory network reactivation from resulting in a cluttered, and therefore conflicting, memory representation of multiple events over the long-term.

Based on the idea that sleep can actively promote a change in the structure of recently encoded memory representations (Gais and Born, 2004; Wagner et al., 2004), we propose, first, that selective memory reactivation during sleep entails the reactivation of associated events (i.e., events that share features) acquired during awake time. Second, that this may lead the brain to adaptively regulate whether these associated memories are strengthened or pruned on the basis of their relative associative strength to the shared element. Specifically, sleep memory reactivation would promote the stabilization of interrelated memory networks by strengthening strongly associated memories and weakening weakly associated memory representations. In this way, memory reactivation during sleep would prevent the consolidation of cluttered memory networks, derived from the inclusion of weakly associated memories that may likely be related to unreliable events in the memory network (Kim et al., 2014).

The notion that selective reactivation of an event can lead either to a strengthening or weakening of associated memories is supported by previous empirical and computational research (Norman et al., 2006, 2007; Newman and Norman, 2010; Detre et al., 2013) on the bases of representational analysis of neuroimaging data and behavioral measures. In these studies, memories associated with a given memory cue are reactivated and those with moderate levels of strength are weakened, whereas those with greater memory strength are strengthened. Thus, in the context of the simultaneous activation of overlapping representations, such a mechanism would sharpen the contrast between strongly and less-strongly associated memories. This, in turn, would adaptively reduce the degree of interference from unreliable associations in subsequent retrieval attempts. Although predictions derived from this model have never been examined during sleep, previous findings using awake implicit (Newman and Norman, 2010; Kim et al., 2014) and explicit (Lewis-Peacock and Norman, 2014; Detre et al., 2013) tasks have revealed that this mechanism well explains how the differential strength of associated memory activation affects memory performance.

To test this proposal in the context of system-level sleep memory consolidation, we developed a paradigm that allowed us to create overlapping memories, manipulate their associative memory strength, and then reactivate them selectively during sleep. We predicted that strong associated memories would benefit from target memory reactivation whereas weak associated ones would be actively forgotten.

\section{Materials and Methods}

\section{Participants}

Participants were college undergraduate students who had normal or corrected-to-normal vision and hearing. We excluded from the study those participants reporting drug consumption or known neurological, psychiatric, or sleep disorders (i.e., atypical sleep patterns like insomnia and frequent awakening at night) based on the Athens Insomnia Scale. All the participants included in the study reported no history of medical, neurological, or psychiatric disorders, and no drug consumption. All subjects were volunteers, gave written informed consent, consented to publication, and received financial compensation for their participation in this study. The study was approved by the Ethics Committee of the University of Barcelona.

Sleep reactivation experiment. A total of 92 college students (mean age $=23.2$ years old, $\mathrm{SD}=3.3 ; 65$ women) participated in the study. We excluded from the analysis those participants who could not achieve stable sleep (i.e., did not reach phase 2 for $>10 \mathrm{~min}$ in the first $30 \mathrm{~min}$ of the sleep session; $n=13$ ), recalled target sounds in the awareness test $(n=20)$, or woke up before completing 34 rounds of the reactivation protocol $(n=6)$. Data from three participants were eliminated due to technical problems with EEG recording. A final sample of 50 participants ( $n=22$ in the contiguous group; $n=28$ in the delay group) was included in the data analysis.

Control Experiment 1: testing memories before sleep. Forty participants ( mean age $=22.5$ years old, $\mathrm{SD}=3.8 ; 29$ women) that fulfilled the same inclusion criteria as participants in the nap group were recruited for Control Experiment 1 ( $n=20$ in the contiguous group; $n=20$ in the delay group).

Control Experiment 2: target memory reactivation during awake. Eighteen participants (mean age $=22.3$ years old, $\mathrm{SD}=3.8 ; 13$ women) that fulfilled the same inclusion criteria as participants in the nap group were recruited for Control Experiment 2.

\section{Experimental procedure}

Overview of the procedure. In this learning paradigm all participants learned two different sets of object-location pairs, X1-X2 and X1-X3 locations, that overlapped in their first card location (X1; Fig. 1).

During the first encoding phase, participants learned 15 locations of pairs of identical cards placed in different positions (X1-X2) within a grid. This was followed by a second memory encoding (X1-X3) phase in which the first card of each pair was presented in the same position (X1; i.e., the overlapping element) but the second card was presented in a different position (X3). Thus, the task required participants to form two distinct sets of memory traces which shared a common element (X1).

To address the extent to which sleep memory reactivation differentially strengthened or weakened overlapping memories as a function of their associative memory strength with the cue (X1) before sleep, we performed a simple manipulation. We changed the delay between the acquisition of the X1-X2 memories and the rest of the phases of the experiment (i.e., X1-X3 encoding, sleep nap, and recall test). One group of participants had no delay, and right after completing the X1-X2 phase proceeded with the rest of the experiment (contiguous group). The other group waited $3 \mathrm{~h}$ before the $\mathrm{X} 1-\mathrm{X} 3$ encoding task (delayed group). In line with the notion that memory strength decays as a function of time (Hardt et al., 2013), X2 memory locations in the delay group should be less strongly associated with the X1 cue after encoding X1-X3 than in the contiguous group. In the current experiment, memory strength was operationally defined behaviorally as its likelihood of being recalled with an overlapping cue. Consequently, X2 memory locations should be less accessible to participants from the delay group in a recall test.

Control Experiment 1: testing memories before sleep. To test whether overlapping memories would differ in their associative memory strength as a consequence of a temporal delay between the two encoding tasks, we ran a separate behavioral control experiment. In it, we asked two groups of participants to recall X2 item location given X1 item on the screen right after the X1-X3 encoding phase, corresponding to the moment that 
ENCODING

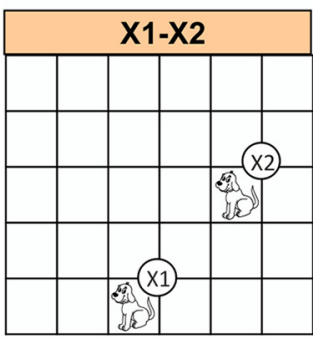

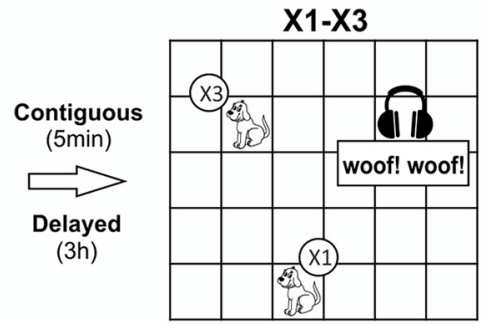

SLEEP

REACTIVATION

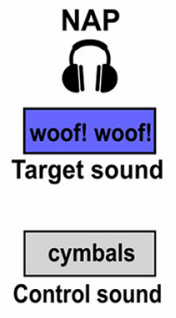

TEST

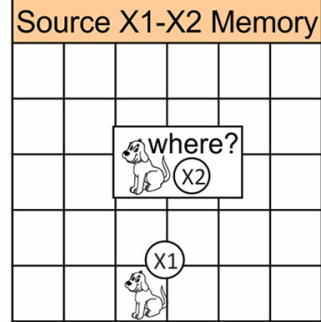

Figure 1. Experimental design and source memory recollection for X1-X2 memories. Participants encoded locations of 15 pairs of identical cards (X1-X2 encoding), followed by a second encoding (X1-X3) in which the first card of each pair was presented in the same location but the second card was presented in a different one. During X1-X3 encoding, card pair locations were associated with 15 distinctive sounds (target sounds). The delay group waited $3 \mathrm{~h}$ before encoding X1-X3 memories, whereas the contiguous group encoded them after 5 min. In a subsequent nap, the nap group was presented with seven target sounds interleaved with a control sound (new sound). Sounds were presented five times each during non-REM sleep. Upon waking, participants were presented with the first card of each pair placed in the grid (X1) and were asked to recollect the matching card located at X2, learned during the first encoding (source X1-X2 memory). In Control Experiment 1, participants encoded only half of the associations during X1-X3 learning and no sounds were presented. These participants did not nap and the source memory test was performed right after encoding X1-X3.

precedes sleep in our main experiment. To also assess the level of interference of X1-X3 association in X1-X2 memories at a within-subject level, participants learned only half of the X1-X3 associations (7 or 8, counterbalanced across subjects). Card pairs presented during X1-X3 encoding were controlled so that they were equally representative of each semantic category.

Sleep reactivation experiment. Critically, in this group encoding of $\mathrm{X} 1-\mathrm{X} 3$ memories was cued with a distinctive sound that was presented in association with each pair of cards. Following previous studies (Rudoy et al., 2009; Fuentemilla et al., 2013; Oudiette et al., 2013), half of these sounds were presented again during a subsequent NREM sleep stage, whereas participants napped after the second encoding phase. In previous studies, this approach, termed targeted memory reactivation (TMR), has been shown to be suitable to investigating the effects of memory reactivation at the within-subject level (Oudiette and Paller, 2013). This experimental approach has the advantage of addressing the role of memory reactivation during sleep in a single-day experimental session in a within-level design. It fits well to previous literature emphasizing that the impact of memory reactivation on memory consolidation occurred during initial periods of sleep (Bendor and Wilson, 2012). However, it has the disadvantage that the participants' sleep is inherently less comfortable than a standard night sleep in a bed. This disadvantage ultimately impacts on the proportion of participants that need to be excluded from the study because of sudden awakenings during the nap session (Participants, Sleep reactivation experiment). In contrast to previous research using TMR experimental designs, we assessed here whether the presentation during sleep of sound cues strongly linked to X1-X3 memories influenced participants' ability to remember their overlapping memory $\mathrm{X} 1-\mathrm{X} 2$. Thus, as in the control experiment, but this time upon awakening, we asked participants to recall each X2 item location given each X1 item on the screen. Here, participants' ability to correctly recall X1-X2 memories as a function of whether they were associated with $\mathrm{X} 1-\mathrm{X} 3$ memories whose sound was presented during sleep could be attributed to off-line memory consolidation. This provides a behavioral measure of how sleep memory reactivation affects the consolidation of overlapping memories.

Stimuli. Fifteen different images with dimensions of $4.5 \times 4.5 \mathrm{~cm}$ equivalent to the dimensions of a cell in a $5 \times 6$ cell grid were displayed. Images were presented on a 19 inch computer monitor placed $70 \mathrm{~cm}$ away from the participant. All 15 distinctive auditory cues were easily recognizable realistic sounds of animals, musical instruments, and means of transportation (Table 1). Sounds had a duration ranging from 1 to $1.5 \mathrm{~s}$ and were presented continuously and repeatedly through headphones for $3 \mathrm{~s}$ each, starting with the presentation of the first image of the pair.

X1-X2 encoding. Participants learned a visuospatial location task of 15 pairs of cards (Diekelmann et al., 2011) depicting animals, musical in-
Table 1. Description of images and corresponding associated sounds used in the experimental protocol

\begin{tabular}{ll}
\hline Image & Sound \\
\hline Dog & "Woof-woof" \\
Sheep & "Baa-baa" \\
Rooster & "Cock-d-doodle-doo" \\
Cow & "Moo-moo" \\
Cat & "Meow-meow" \\
Harp & Sound of a harp chord \\
Guitar & Sound of a guitar chord \\
Saxophone & Sequence of notes on a saxophone \\
Drum & Percussion on a drum \\
Piano & Sequence of notes on a piano \\
Train & Train horn \\
Ship & Ship horn \\
Helicopter & Helicopter noise \\
Car & Car engine noise \\
Plane & Plane noise \\
\hline
\end{tabular}

struments, and means of transportation. First, every card pair location (X1-X2) was randomly revealed twice and participants were asked to memorize the locations. Card pair presentations always began with the presentation of the first card (X1) followed $1 \mathrm{~s}$ later by the appearance of its matching card (X2) presented for $2 \mathrm{~s}$ before both cards disappeared. The intertrial interval between card pair presentations was $2 \mathrm{~s}$. Right after cards were revealed, participants were presented with the first card of each pair (X1) and were asked to find the matching card (X2) by clicking with the mouse on the correct location. Participants were asked to withhold their response until the mouse cursor appeared $(2 \mathrm{~s}$ after the first card onset). After responding, participants received a $3 \mathrm{~s}$ feedback of the correct location. Card pair presentations were organized in blocks in which each pair was presented once, so each block always contained all 15 card pairs. The order of presentation of the card pairs was randomized for each block. The encoding phase concluded when participants correctly indicated the location of at least 14 matching cards within a block, that is, $>90 \%$ of responses correct (Diekelmann et al., 2011).

$X 1-X 3$ encoding. Participants were requested to learn different new locations for the same card pairs presented in the previous phase. They were presented with the same grid and learning was conducted following the same procedure used for X1-X2 encoding. The first card of each pair was presented in the same location as in the initial encoding (X1), functioning as the overlapping element between both encoding tasks. However, the second card of each pair was located in a different location (X3) and a specific distinctive sound accompanied each card pair presentation throughout the encoding phase (e.g., a pair of dogs with a barking sound; 
Table 1). Therefore each card pair new location (X1-X3) was associated with a specific sound. The same learning criterion was used in this phase.

Contiguous group. After encoding memory X1-X2, participants were told that a new encoding phase was about to begin. Participants were told that they could relax for $5 \mathrm{~min}$ and continue with the next encoding session.

Delayed group. The same experimental design and procedure was implemented and conducted in the same manner as in the continuous encoding group. However this time, participants encoded $\mathrm{X} 1-\mathrm{X} 3$ positions $3 \mathrm{~h}$ after encoding $\mathrm{X} 1-\mathrm{X} 2$ positions (Fig. $1 ; \mathrm{M}=147.8, \mathrm{SD}=16.61$ ). During this interval, to prevent rehearsing of previous locations, participants watched a movie in a separate room. To reduce new encodings while watching the movie, participants had to choose the movie they knew best from a selection of seven popular movies. After the movie, participants completed the X1-X3 encoding session and the EEG cap was positioned before the nap session began.

Nap session. To promote sleeping during the study, experiments were conducted after lunchtime (starting between 2:00 P.M. and 3:00 P.M.) and all participants were asked to reduce their regular hours of sleep by $25 \%$ during the night preceding the experiment. Participants did not know that they were going to be trained or tested for any memory, at any time. Participants napped in the same sound-attenuated room where encoding took place. Following the experimental setting used in previous studies (Rudoy et al., 2009), the room was dimly lit and participants sat in a $45^{\circ}$ reclined chair and were provided with a blanket and a pillow. Sessions lasted $63.81 \mathrm{~min}(\mathrm{SD}=11.06)$ during which participants slept for an average of $48.46 \mathrm{~min}(\mathrm{SD}=14.34)$. As in previous studies using auditory cues during sleep (Rudoy et al., 2009; Fuentemilla et al., 2013), to prevent abrupt changes during the reactivation protocol, we set a constant background audio input consisting of white noise $(35 \mathrm{~dB}$ sound pressure level). We also included a repetitive sound (i.e., birds) throughout the sleep session. The repetitive sound was presented right from the beginning of the sleep session (even when the participants were still awake) and served to ensure that the sudden appearance of an audio input would not awaken the participants or disrupt the ongoing sleep stage architecture.

The memory reactivation protocol began $22.04 \mathrm{~min}(\mathrm{SD}=7.54 \mathrm{~min})$ after the beginning of the sleep session when stage 2 of sleep was detected (monitored on the basis of standard sleep scoring with $\mathrm{Fz}$ electrode (Mölle et al., 2002). The memory reactivation protocol lasted 14 min and included the presentation of seven randomly selected auditory stimuli (target sounds) that were previously associated with memory X1-X3. Participants from the continuous and delayed groups spent an average of $16.41 \%(\mathrm{SD}=32.37)$ and $34 \%(\mathrm{SD}=29.97)$ of the reactivation protocol time, respectively, in slow-wave sleep. The presentation of target sounds was alternated with the presentation of a control sound (i.e., cymbals) that had not been presented previously to the participants. This control sound was later used to study the EEG neural activity in response to auditory cues triggering memory reactivation (target sounds), as opposed to neural responses elicited by auditory input without associated memory content. Reactivation was interrupted if signs of arousal or awakening were detected in the EEG. The stimulation resumed after stable non-REM sleep was reestablished. The memory reactivation protocol involved the presentation of sounds organized in rounds that comprised three sounds in the following order: a $3 \mathrm{~s}$ target sound, a $3 \mathrm{~s}$ control sound, and a $3 \mathrm{~s}$ repetitive sound with an interval of $5 \mathrm{~s}$ of only white noise between sounds. Sounds were presented from the start of the sleep session and were embedded within the background white noise. We delivered 35 rounds so that each of the seven target sounds was presented five times (only one participant, included in the analyses, did not complete the last round). White noise was presented throughout the sleep session and stimuli were embedded in it with the same intensity. Afterward, participants continued sleeping with white noise until naturally waking up. Participants that did not wake up naturally $(n=2)$ were woken up by the experimenter after completing $80 \mathrm{~min}$ from the beginning to the end of the sleep session. After waking up, and to break sleep inertia, participants were encouraged to talk and to have a snack and a beverage before starting the next task (Source X1-X2 memory test).
Source X1-X2 memory test. After a further 5 min delay after the sleep session, spatial locations for $\mathrm{X} 1-\mathrm{X} 2$ memories were tested. As in the pre-nap encoding phase, the overlapping card from each pair was placed in the grid (X1), and participants were asked to recall the position of the matching card learned during the $\mathrm{X} 1-\mathrm{X} 2$ encoding phase. This time, no cards were revealed after the response, and the next trial started immediately without any feedback.

Recognition memory test. Participants were presented with the overlapping card from each pair placed in the grid (X1). After $2 \mathrm{~s}$, the second card appeared either at X2 or in a new location. Participants were requested to report whether the second card location matched the one presented during X1-X2 encoding. Participants responded by pressing "c" ("correct") or "I" ("incorrect") on the keyboard.

Awareness test. Next, participants were asked to recall any sound that they might have heard or noticed during their nap. The recall test was followed by a memory recognition test. The recognition test allowed participants to recognize those sounds that they might not have been able to recall previously. Hence, all 15 sounds were presented in random order and participants were asked to indicate those they intuited as having been presented during reactivation.

Control Experiment 2: targeted memory reactivation during awake. We conducted an additional separate control experiment to test the possibility that the observed behavioral memory effects were not sleep-specific. A similar experimental protocol was thus implemented as in the contiguous group, but participants remained awake while sound cues were presented. The experimental design and procedure described for the encoding session, the $\mathrm{X} 1-\mathrm{X} 2$ source memory test, and the awareness test, was implemented in the same manner as in the sleep reactivation experiment. The targeted memory reactivation session, in contrast, took place while participants were awake. To match the first minutes before the nap group fell asleep and started the reactivation protocol, participants were instructed to close their eyes for $15 \mathrm{~min}$ and not fall asleep while listening to the background noise which remained present again throughout the entire session (white noise and repetitive sound, as in the nap group). Whenever signs of sleepiness in the EEG recording were detected, participants were verbally reminded to stay awake. After this 15 min interval they were engaged in an N-back working memory task. Digits were visually presented in succession in the middle of the screen. They were displayed for 500 milliseconds with a $2 \mathrm{~s}$ intertrial interval. After each presentation, participants were asked to indicate if the current digit and the one before the previous (2-back task) were the "same" (either both odd or even) or "different" (one odd and one even) by pressing the keyboard letter " $\mathrm{z}$ " (to indicate same) or " $\mathrm{m}$ " (to indicate different). Participants had unlimited time to answer and received a $500 \mathrm{~ms}$ feedback after incorrect responses: "incorrect answer, pay more attention next time." Participants were trained in the task for $\sim 5 \mathrm{~min}$ while listening to the background noise (white noise and repetitive sound). They then performed this task for 20 min without feedback, while being exposed to the memory reactivation protocol. Sounds were presented in the same succession as in the nap group (i.e., target, control, and then repetitive sound) and were separated by a 5 s intertrial interval. All participants completed the reactivation protocol and therefore received the same number of sound reactivations as the nap group. Each of these sounds was delivered after digit presentation and before the question appeared. This way, we ensured that participants were engaged in the distracting task at the moment the sounds were presented. Therefore, participants were prevented from allocating attention to the sounds and rehearsing previously learned associations. To match total time between encoding and memory test for the sleep reactivation group, the memory reactivation protocol was followed by a low arousal movie to complete the $65 \mathrm{~min}$ from the beginning of the nap session. Next, participants performed the awareness test following the same procedure used in the sleep reactivation group.

\section{Experimental design and statistical analysis}

In the current design we examined the extent to which sleep memory reactivation differentially strengthened or weakened overlapping memories as a function of their associative memory strength with the cue (X1) before sleep. To address this central question in the behavioral data, we 
examined memory accuracy for $\mathrm{X} 1-\mathrm{X} 2$ memories at Source $\mathrm{X} 1-\mathrm{X} 2$ memory test.

Memory recollection accuracy was calculated as the correct responses (hits) divided by the total number of reactivated $(n=7)$ or nonreactivated associated memories $(n=8)$. Memory recollection accuracy for X1-X2 locations in Control Experiment 1 was calculated as the correct responses (hits) divided by the total number of interfered ( 7 or 8 ) or non-interfered associated memories (7 or 8). We also looked for memory intrusions calculated as individual responses at the $\mathrm{X} 3$ positions during $\mathrm{X} 1-\mathrm{X} 2$ memory test, divided by the total number of errors.

Repeated-measures ANOVA and Wilcoxon match-pairs signed rank test were used to identify differential memory accuracy between groups and experimental conditions. Alpha was set at 0.05 . Effect sizes $R$, partial $\eta$-squared, and Cohen's $d$ were reported as appropriate.

\section{EEG data acquisition and analysis}

EEG recording. EEG data were recorded only for the sleep reactivation groups. EEG was recorded using a Neuroscan system. Tin electrodes were mounted in an electro-cap (Electro-Cap) and were located at 29 standard positions (Fp1/2, Fz, F7/8, F3/4, Fc1, Fcz, Fc2 Fc5/6, Cz, C3/4, T3/4, $\mathrm{Cp} 1 / 2, \mathrm{Cp} 5 / 6, \mathrm{Pz}, \mathrm{P} 3 / 4, \mathrm{~T} 5 / 6, \mathrm{PO} 1 / 2, \mathrm{Oz})$. Vertical eye movements were monitored with an electrode at the infraorbital ridge of the right eye. Electrode impedances were kept $<3 \mathrm{k} \Omega$. Electrophysiological data were sampled at $250 \mathrm{~Hz}$, notch-filtered (at $50 \mathrm{~Hz}$ ), bandpass-filtered with a range of $0.05-50 \mathrm{~Hz}$, and referenced to the mean of the right and left mastoids. The same procedures were applied for the delayed group but EEG was recorded using 10 central electrodes (Fz, F3/4, Fcz, Cz, C3/4, Pz, $\mathrm{P} 3 / 4)$.

Spindles and slow oscillation analysis. During the reactivation protocol spindles and slow oscillations (SOs) were quantified and measured in amplitude and duration for each subject. We used a MATLAB (2008b, Mathworks) implemented detection algorithm (Fuentemilla et al., 2013) and applied it to the Fz channel EEG data, where spindle and SO are pronounced (Mölle et al., 2002). To identify spindles, the EEG signal was zero-phase bandpass filtered between 11 and $15 \mathrm{~Hz}$ with a fourth-order bandpass filter using a linear finite impulse response (FIR) filter, and its envelope was computed. The instantaneous amplitude and envelope during NREM were computed via the Hilbert transform. Whenever the envelope crossed an upper threshold of $2.5 \mathrm{SD}$ over the average envelope, a potential spindle was identified. A start/end threshold was set at a value of the mean plus $1 \mathrm{SD}$. Only events with durations between 0.4 and $4 \mathrm{~s}$ were further considered. To identify SOs, and to exclude the effects arising from neural generators in the delta band (i.e., $1-3 \mathrm{~Hz}$ ), EEG data were bandpass filtered (FIR) between 0.4 and $1 \mathrm{~Hz}$. Then, the largest negative half-waves were selected from slow-wave sleep (stages 3 and 4) using a threshold procedure applied to the SO signal. The negative half-wave detection was chosen for the analysis due to the high degree of variability in the positive signal deflections compared with the stability of the negative deflections (Riedner et al., 2007). The peak time of a half-wave found was used if the following criteria were fulfilled: (1) the two ends of the half-wave were two succeeding zero-crossings of the slow wave separated from each other by $0.25-1.5 \mathrm{~s}$ (Mölle et al., 2002) and (2) the peak amplitude between both zero-crossings exceeded a threshold of at least 2 SD multiplied by the averaged amplitude.

Time-frequency analyses. Time-frequency analysis was performed on a single trial basis, with epochs of 4.1 s locked to the onset of each sound and seven-cycle complex Morlet wavelets. An additional padding window of $2 \mathrm{~s}$ before and after the epoch was used to eliminate edge effects. Frequencies from 1 to $40 \mathrm{~Hz}$, using a step of $1 \mathrm{~Hz}$, were selected. The frequency power was calculated for each frequency and time point. The time period from -100 to $0 \mathrm{~ms}$ from the onset of the stimulus was used as baseline. For each participant, the average of the trials of the two conditions (target sounds and control sounds) was then calculated. The proportion of change in power of each data point was calculated dividing the values for each data point by the mean power of their corresponding frequency during the baseline period. The two conditions were compared using a cluster-based permutation analysis (Ngo et al., 2013). First, paired $t$ tests were performed for all points of the time-frequency matrix. In a second step, clusters in the Fz electrode that had $>200$ adjacent points, either in time or frequency dimensions, with a difference between conditions of $p<0.01$, were detected. Using the value of the cluster with the largest sum of absolute $t$ values, we calculated the permutation distribution, using 10,000 random samples. The test was two-way and it had a critical $\alpha$ value of 0.05 .

\section{Results}

Memory performance before sleep (Control Experiment 1)

In the recall test, the delayed group showed overall a poorer ability for X1-X2 memories compared with the contiguous group (main effect of group: $F_{(1,38)}=6.94, p=0.01, \eta^{2}=0.15$; Fig. $2 A$ ). Such differences in memory recollection were independent of interference effects triggered by encoding X1-X3 events that overlapped in content with X1-X2 events (main effect of interference: $F_{(1,38)}=20.27, p<0.001, \eta^{2}=0.35$; but no significant interference $\times$ group interaction: $F_{(1,38)}=0.33, p=0.57, \eta^{2}=$ $0.009)$. In addition, in the recognition test, we found that noninterfered associations were better recognized than interfered ones (recognition memory test; main effect of interference: $\left.F_{(1,38)}=10.10, p<0.005, \eta_{p}^{2}=0.21\right)$. However, we did not find an overall differential pattern of memory accuracy between groups $\left(F_{(1,38)}=1.60, p<0.21, \eta_{p}^{2}=0.04\right)$. This suggests that differences observed in participants' ability to retrieve X2 memory locations that were interfered with and those that were not interfered with are susceptible to the retrieval processes engaged during the test (i.e., recall and recognition). The ability to retrieve weaker memories associated to X1 cue is heavily impaired during recall but not during recognition tasks (Haist et al., 1992). Finally, corroborating previous recall findings, we found a nonsignificant interference $\times$ group interaction effect $\left(F_{(1,38)}=1.03, p<0.31\right.$, $\eta_{p}^{2}=0.02$ ), thereby providing evidence of the null influence of delay between encoding sessions as to how novel encoding interferes previously stored memories.

\section{The impact of targeted memory reactivation during sleep in a} subsequent memory test (sleep reactivation experiment)

A repeated-measures ANOVA revealed better overall performance for the contiguous group than the delay group (main effect of group: $F_{(1,48)}=14.56, p<0.001, \eta^{2}=0.23$ ). More interestingly, we found a significant group $\times$ reactivation interaction $\left(F_{(1,48)}=6.181, p=0.01, \eta^{2}=0.11\right)$ but no main effect of reactivation $\left(F_{(1,48)}=0.65, p=0.42, \eta^{2}=0.01\right)$. Thus, participants from the contiguous group were more accurate in recalling associated X1-X2 memories linked to sound cues that were presented during sleep than the associated X1-X2 memories linked to sound cues that were $\operatorname{not}(Z=1.96, p=0.04$ Wilcoxon matchpairs signed rank test; effect size of $r=0.29$; Fig. $2 B$ ). Notably, the opposite pattern of results was observed in the delayed group. Thus, participants from that group showed lower memory performance in recalling X1-X2 memories linked to sound cues presented during sleep compared with $\mathrm{X} 1-\mathrm{X} 2$ memories linked to sounds never presented during sleep $(Z=-2.028, p=0.04, r=$ $0.27)$. No significant effects were found for memory intrusions (contiguous group: $\mathrm{M}=0.47, \mathrm{SD}=0.22$; delayed group: $\mathrm{M}=$ $0.54, \mathrm{SD}=0.13)$ between groups $\left(F_{(1,48)}=0.66, p=0.42, \eta_{p}^{2}=\right.$ $0.01)$ or between reactivated and non-reactivated associated card pairs $\left(F_{(1,48)}=0.01, p=0.91, \eta_{p}^{2}<0.01\right.$; group $\times$ reactivation interaction: $\left.F_{(1,48)}=1.76, p=0.19, \eta_{p}^{2}=0.03\right)$. Finally, in contrast with the recall test, effects triggered by the reactivation manipulation were no longer observed in participants' memory performance in the following recognition test (main effect of memory reactivation: $F_{(1,48)}=0.35, p=0.56, \eta_{p}^{2}=0.007$; group $\times$ reactivation: $\left.F_{(1,48)}<0.01, p=0.936, \eta_{p}^{2}<0.01\right)$. However, the overall recognition memory was greater for the contig- 


\section{A Control experiment}

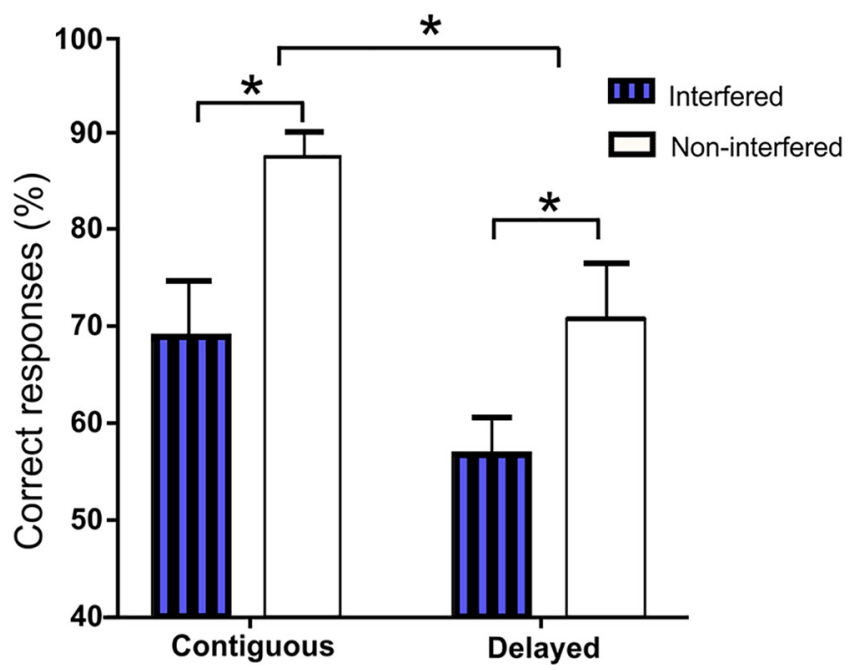

\section{B Sleep reactivation experiment}

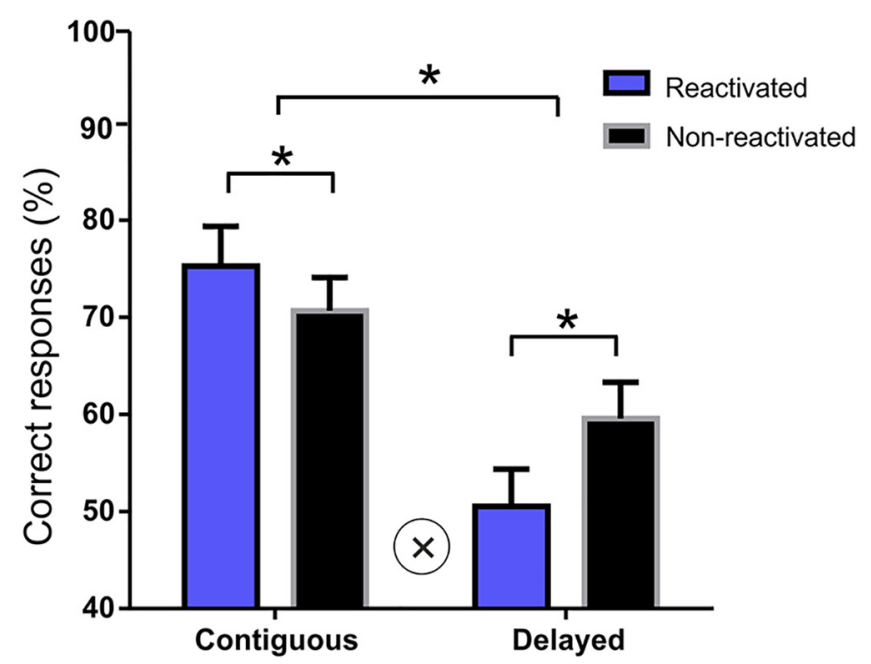

Figure 2. Source memory recollection for X1-X2 memories. A, Bars show the average percentage of correct responses across participants for the contiguous and delayed groups in Control Experiment 1, in which memories were tested right before sleep. Compared with the contiguous encoding, delayed encoding decreased overall memory recollection independently of the effects of interference. Interference effects were equivalent in the two groups and were selective for those associations that were learned again during X1-X3 encoding. $\boldsymbol{B}$, Bars show the average percentage of correct responses across participants in the sleep reactivation experiment. Contiguous encoding enhanced memory recollection for X1-X2 memories that overlapped with reactivated X1-X3 memories. The opposite effect was observed in the delayed group. X1-X2 associations that overlapped with reactivated X1-X3 memories were less well remembered than those that overlapped with non-reactivated $X 1-X 3$ memories. ${ }^{*} p<0.05$. Error bars represent SEM. $X=$ significant ANOVA interaction.

uous in comparison with the delayed group $\left(F_{(1,48)}=16.11, p<\right.$ $0.001, \eta_{p}^{2}=0.25$ ).

Target memory re-exposure during awake does not influence memory performance in a subsequent test (Control Experiment 2) Here, we tested the possibility that the observed behavioral memory effects triggered by target reactivation were not sleep-specific. A similar experimental protocol as in the continuous group was thus implemented, but participants remained engaged in an attention-demanding task (i.e., N-back) while sound cues were presented. At the end of the experiment, participants correctly recognized an average of $90 \%(\mathrm{SD}=6)$ of the reactivated sounds and recalled only $30 \%(S D=19)$ of them, suggesting that the N-back task successfully prevented a deep encoding of the sounds. The general memory performance was numerically lower than for the Sleep reactivation group (sleep reactivation group: $\mathrm{M}=75.11 \%, \mathrm{SD}=15.64$; wake group: $\mathrm{M}=61.74 \%$, $\mathrm{SD}=21.29)$ yet this difference did not reach statistical significance (Mann-Whitney nonparametric test unpaired, $Z=-1.65$, $p=0.09, r=-0.25)$. In contrast to the sleep reactivation group, here similar recall performance was observed for X1-X2 memories associated with $\mathrm{X} 1-\mathrm{X} 3$ memories whose sounds were reexposed $(\mathrm{M}=63.16 \%, \mathrm{SD}=24.73)$ compared with those associated with memories whose sounds were not re-exposed $(\mathrm{M}=60.79 \%, \mathrm{SD}=25.75 ; Z=0.48, p=0.63, r=0.27)$, indicating that the behavioral effects of targeted memory reactivation were specific to sleep.

\section{No semantic facilitation of targeted memory reactivation during sleep}

According to recent findings, memory benefits of targeted memory reactivation may be extensive, under specific circumstances, with memories that belong to the same semantic category (Oudiette et al., 2013). To rule out this possibility in our experiment, we examined whether the selected memories to be reactivated and not reactivated during sleep were equally distributed within the three semantic categories from which the 15 pair objects used in our study were taken (animals, musical instruments, and means of transportation). To this end, we calculated the mean number of items from each category that were selected for reactivation and then performed a binomial probability test. This analysis revealed that the number of exemplars from each category did not significantly differ between conditions (reactivated and non-reactivated; Animals, continuous group: $\mathrm{M}(\mathrm{R})=55.4 \%$, $\mathrm{SD}=18$, binomial probability test, $p=0.5$; delay group: $\mathrm{M}(\mathrm{R})=$ $38.57 \%, \mathrm{SD}=19.57$, binomial probability test, $p=0.19$; Musical instruments, contiguous group: $\mathrm{M}(\mathrm{R})=48.2 \%, \mathrm{SD}=19.7, p=$ 0.5 ; delayed group: $\mathrm{M}(\mathrm{R})=51.42 \%, \mathrm{SD}=18.4, p=0.5$; Means of transportation, continuous group: $\mathrm{M}(\mathrm{R})=36.4 \%, \mathrm{SD}=17.7$, $p=0.19$; delayed group: $\mathrm{M}(\mathrm{R})=50 \%, \mathrm{SD}=17.63, p=0.5)$. In addition, we found no correlation between the number of times each category was reactivated and the accuracy for nonreactivated items from the same category (Animals, contiguous: $r_{(20)}=0.02, p=0.90$; delayed: $\left.r_{(28)}<0.01, p=0.99\right)$; Instruments, contiguous: $r_{(20)}=0.03, p=0.82$; delayed: $r_{(28)}=0.14$, $p=0.48$; Means of transportation, contiguous: $r_{(20)}=0.02, p=$ 0.92; delayed: $\left.r_{(28)}=0.03, p=0.84\right)$. These results show that reactivated associations did not trigger semantic facilitation over non-reactivated memories.

Spindles and SOs induced by targeted memory reactivation Participants in the continuous and delayed groups presented a mean of $4.04(\mathrm{SD}=0.23)$ and $4.17(\mathrm{SD}=0.45)$ spindles per second and $0.10(\mathrm{SD}=0.01)$ and $0.11(\mathrm{SD}=0.04)$ SOs per second, respectively, during the reactivation protocol.

Given the opposite pattern of behavioral results in the two sleep groups we sought to examine whether different neural mechanisms could be involved during memory reactivation. Thus, in this first analysis, we examined whether target sound cues elicited changes in the pattern of SO and spindle activity and compared the results to those $\mathrm{SO}$ and spindle patterns associated with the presentation of control novel sounds (i.e., sounds that 


\section{Contiguous group}
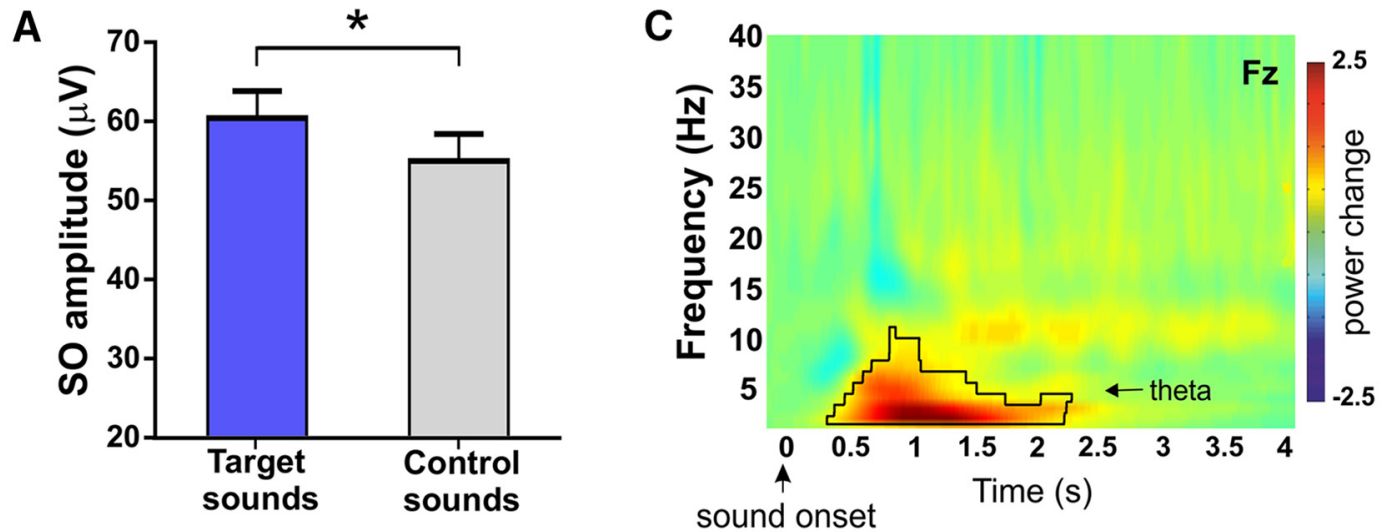

E

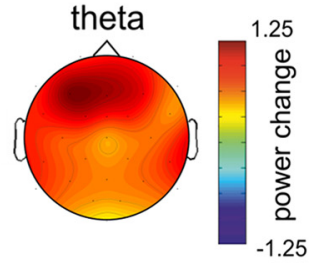

\section{Delayed group}
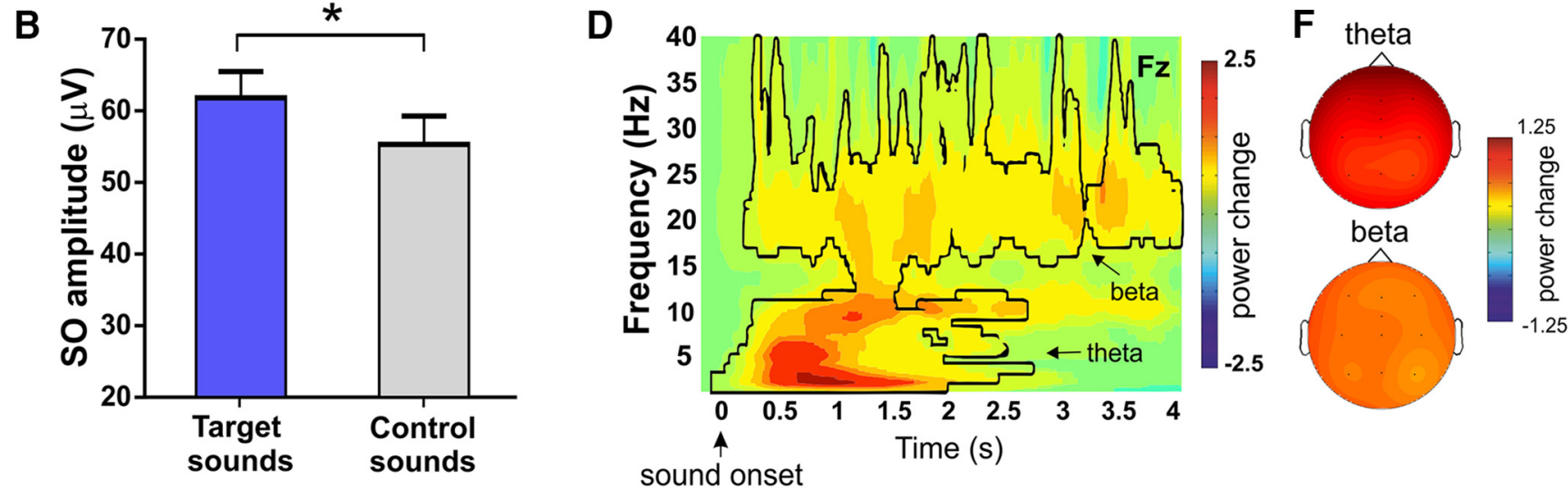

Figure 3. Neurophysiological responses to target sound presentation during sleep (sleep reactivation experiment). $A, B$, Average amplitude of the $50 \mathrm{~s}(0.1-4 \mathrm{~Hz})$ during $8 \mathrm{~s}$ from the onset of target and control sound presentation for the contiguous $(\boldsymbol{A})$ and delayed $(\boldsymbol{B})$ groups. ${ }^{*} p<0.05$. Error bars represent SEM. $\boldsymbol{C}, \boldsymbol{D}$, EEG spectral power differences between target sounds (associated with X1-X3 memory) and control sounds during sleep for the contiguous $(\boldsymbol{C})$ and delayed $(\boldsymbol{D})$ groups. $p<0.05$, cluster-based permutation test for theta power; $p<0.001$, cluster-based permutation test for beta power. Significant clusters are delimited with a black line and displayed for the Fz electrode. The legend adjacent to the EEG spectral power graphs represents the power change proportion with respect to baseline. E, F, Scalp topography depicting the significant theta and beta cluster found when comparing the power responses between target and control sounds for the contiguous $(\boldsymbol{E})$ and delayed $(\boldsymbol{F})$ groups. The legend adjacent to the topography represents the power change proportion with respect to baseline.

were never presented during encoding and that therefore were not linked to any memory) interleaved during NREM sleep.

The analysis of the spindle activity showed a greater number of spindles elicited by target sounds compared with control sounds $\left(F_{(1,45)}=4.58, p=0.03, \eta_{p}^{2}=0.09\right)$, and also showed that this increment was similar in the two groups (main effect of group: $F_{(1,45)}=.03 ; p=0.85 ; \eta_{p}^{2}<0.01$; interaction: $F_{(1,45)}=0.43$; $\left.p=0.51 ; \eta_{p}^{2}=0.01\right)$. In addition, we did not find any statistically significant effects in terms of spindle amplitude (group: $F_{(1,45)}=0.48, p=0.49, \eta_{p}^{2}=0.01$; sound type: $F_{(1,45)}=1.32, p=$ $0.25, \eta_{p}^{2}=0.02$; interaction: $\left.F_{(1,45)}=0.08 ; p=0.77 ; \eta_{p}^{2}<0.01\right)$ or length (group: $F_{(1,45)}<0.01, p=0.93, \eta_{p}^{2}<0.01$; sound type: $F_{(1,45)}=2.9, p=0.09, \eta_{p}^{2}=0.06$; interaction: $F_{(1,45)}=0.40, p=$ $\left.0.52, \eta_{p}^{2}<0.01\right)$

Regarding SOs, we observed significantly greater amounts of SOs elicited during target sound compared with control sounds $\left(F_{(1,45)}=51.83, p<0.001, \eta_{p}^{2}=0.53\right)$ and with greater amplitude $\left(F_{(1,45)}=31.42, p<0.001, \eta_{p}^{2}=0.41\right)$ but not length $\left(F_{(1,45)}=\right.$ 1.6, $p=0.2, \eta_{p}^{2}=0.03$; Fig. $\left.3 A, B\right)$. These effects were similar between groups (SO quantity: $F_{(1,45)}=1.09, p=0.30 ; \eta_{p}^{2}=0.02$; SO amplitude: $F_{(1,45)}=0.03, p=0.86, \eta_{p}^{2}<0.01$; SO length: $\left.F_{(1,45)}<0.01, p=0.99, \eta_{p}^{2}<0.01\right)$ and no interaction was found between group and sound type (target vs control sounds; SO quantity: $F_{(1,45)}=1.67, p=0.20, \eta_{p}^{2}=0.03$; SO amplitude: $F_{(1,45)}=0.27, p=0.60, \eta_{p}^{2}<0.01$; SO length: $F_{(1,45)}=0.76, p=$ $\left.0.38, \eta_{p}^{2}=0.01\right)$. Follow-up paired $t$ test confirmed a significantly greater number and amplitude of SOs during target than during control sound presentations in both the contiguous group (number: $t_{(21)}=3.82, p=0.001, d_{z}=1.33$; amplitude: $\left.t_{(21)}=3.62, p<0.005, d_{z}=0.33\right)$ and the delayed group (number: $t_{(24)}=6.57, p<0.001, d_{z}=1.85$; amplitude: $t_{(24)}=$ 4.33, $\left.p<0.001, d_{z}=0.34\right)$.

Neural oscillatory responses induced by target memory reactivation during sleep

We examined the role of neural oscillations induced by target sound cues during sleep. Brain oscillatory activity, even when acquired from scalp EEG recordings, has been shown to be a 
suitable approach to investigate the differential involvement during learning-related processes (Tort et al., 2009; Düzel et al., 2010). In the context of this study, our working hypothesis was that target sound cues elicited neural oscillatory response patterns that involved different frequency ranges in the contiguous and delayed groups. More specifically, in humans, theta activity (3-8 Hz) has been prevalently linked to successful memory encoding and retrieval during waking (Düzel et al., 2005; Nyhus and Curran, 2010). Interestingly, recent EEG studies have also demonstrated that theta activity indexed successful cued-memory reactivation during sleep (Schreiner and Rasch, 2015; Schreiner et al., 2015). On the other hand, previous EEG findings revealed a consistent increase in the beta band $(15-30 \mathrm{~Hz})$ upon memory competition during awake periods which was predictive of the degree of memory forgetting to competing memories during retrieval stages (Hanslmayr et al., 2012). Thus, in this experiment, we predicted similar theta power changes elicited by target sound cues in both the contiguous and the delayed groups, whereas changes in the beta band would be observed only in the delayed group. Notably, we found that, when compared with control sound, target sounds enhanced theta power between 500 and $2000 \mathrm{~ms}$ after auditory target cue onset (sound type: $F_{(1,45)}=$ 33.44, $p<0.001, \eta_{p}^{2}=0.42$ ), similarly in the two experimental groups (group: $F_{(1,45)}=0.001, p=0.97, \eta_{p}^{2}<0.01$; interaction: $\left.F_{(1,45)}=0.89, p=0.35, \eta_{p}^{2}=0.01\right)$. However, we found that target sounds induced an increased neural oscillatory response at the beta band (sound type: $F_{(1,45)}=30.54, p<0.001, \eta_{p}^{2}=0.40$ ) that was only observed in the delayed group (group $\times$ sound type interaction: $F_{(1,45)}=4.95, p=0.03, \eta_{p}^{2}=0.10$; group: $F_{(1,45)}=$ $0.76, p=0.38, \eta_{p}^{2}=0.01$; Fig. $\left.3 C, D\right)$. A paired $t$ test on theta (averaged over $3-8 \mathrm{~Hz}$ range) and beta (averaged over $15-30 \mathrm{~Hz}$ range) power, averaged from 500 to $2000 \mathrm{~ms}$ at $\mathrm{Fz}$ electrode (selected as a representative electrode from the scalp based on effects depicted in Fig. $3 E, F)$ confirmed that theta increase was similar in the two groups of participants $\left(t_{(45)}=1.26, p=\right.$ $0.21, d z=0.36$ ) but that the beta band power was higher in the delayed as compared with the contiguous group $\left(t_{(45)}=2.31\right.$, $p=0.02, d z=0.67)$.

\section{Neural oscillatory responses induced by target memory} reactivation during sleep are unlikely to be explained by microarousals during sound presentation

Because sound presentations during sleep can normally induce microarousals, two separate sleep EEG experts blind to conditions scored microarousals after sound onset. Microarousals were identified as abrupt changes of frequency of length between 1.5 and $3 \mathrm{~s}$ (on the $\alpha$ and beta, $16-40 \mathrm{~Hz}$, bands; Bonnet et al., 1992; Mathur and Douglas, 1995; Martin et al., 1997). Overall, the two raters coincided in identifying very little number of microarousals (i.e., $<1 \%$ of the trials in each condition) during sound cue presentation across participants (contiguous-target mean number of trials $=0.27$ and 0.36 (first and second rater, respectively); contiguous-control mean $=0.36$ and 0.54 ; delayed-target mean $=$ 0.88 and 0.44 ; delayed-control mean $=0.6$ and 0.36 ). Importantly, the number of microarousals did not differ significantly between target and control sounds in either experimental group [Wilcoxon rank sum test (target vs control): contiguous group: $Z=-0.707$, $p=0.48$ and $Z=-0.911, p=0.36$ (first and second rater, respectively); delayed group: $Z=-1.72, p=0.08$ and $Z=0.633, p=0.53$ ]. In addition, an inter-rater reliability analysis using the intraclass correlation coefficient (ICC) was implemented and showed moderate/ good "absolute" agreement between the two raters which was significantly different from zero in all experimental groups and con- ditions (two-way random-effects model; contiguous group: Target: ICC $=0.79, p<0.01$; Control: $\mathrm{ICC}=0.70, p<0.01$; delayed group: Target: ICC $=0.50, p=0.04$; Control: $\mathrm{ICC}=0.56, p=0.02$ ). Finally, we ran a new analysis including 19 participants (of the 25 participants) who, according to the first rater, showed exactly the same number of microarousals during target and control sounds (i.e., 2 participants with 1 microarousal per sound type, 1 participant with 4 microarousals per sound type, and 16 participants with no microarousals) in the delayed group. Beta increase for target sounds remained significantly higher in the subsample (paired $t$ test: $t_{(18)}=$ $4.45, p<0.001$ ), thereby ruling out the possibility that beta increase after target sounds could be attributed to greater number of microarousals during this auditory stimulation.

\section{Discussion}

The present study provides the first evidence that selective memory reactivation during sleep entails the reactivation of overlapping memory events acquired during awake time. Our findings showed that targeted memory reactivation during sleep could either promote the strengthening or the weakening of the associated memories as a function of whether they were encoded contiguously or delayed, respectively, in a previous awake time.

Our experimental design required participants to learn a set of overlapping events and to encode them either contiguously ( $5 \mathrm{~min}$ ) or delayed $(3 \mathrm{~h})$ in time. Our first control experiment in which memories were tested right after the encoding of the second set of events showed that delay between encoding phases effectively impoverished the ability to recall the first set of encoded events in the delayed encoding group. Thus, confirming that our experimental manipulation led to strongly and less strongly associated memory representations on the basis of their ability to be recalled by the cue. In a second experiment, we show that the reactivation of a subset of memories during subsequent sleep led to a differential recall ability in a later test upon awakening. More concretely, we found an enhanced recall of strongly associated memories (contiguous group) but an increased forgetting for the less strongly associated memories (delayed group) that were reactivated during sleep in a following recall test. Finally, our work also demonstrated the engagement of different neural oscillatory responses in the theta $(3-8 \mathrm{~Hz})$ and beta $(15-30 \mathrm{~Hz})$ range upon memory reactivation during sleep that relates to whether memories would be subsequently remembered or forgotten. These results suggest the recruitment of an additional regulatory neural mechanism for weakening competing associated memories upon reactivation during sleep.

Note, however, that the use of the term "weakening" is not meant to imply that traces are being deleted completely from memory. Rather, we refer to a mnemonic regulation process in which their accessibility is reduced in a graded manner. This graded pattern of accessibility might especially manifest in conditions under which successful retrieval requires a qualitatively greater reinstatement of the event, such as during a recall but not during a recognition task (Haist et al., 1992). Indeed, participants' performance in a subsequent recognition test did not show memory modulations of the associated memories linked to sound cues presented during sleep.

A limitation of our study is the lack of behavioral data about participants' ability to retrieve the association learned in the second encoding session (i.e., X1-X3). This test was not conducted to avoid possible bias to X1-X2 retrieval performance on the test, which was the main focus of the study. Indeed, previous studies have shown that the act of retrieval influences how associated memories are later retrieved (Anderson et al., 1994). Noteworthy, 
future studies testing $\mathrm{X} 1-\mathrm{X} 3$ as well as $\mathrm{X} 1-\mathrm{X} 2$ memories in a between-subject design would help assess whether the theoretical account tested here was indeed specific to overlapping associations and their associative strength with the reactivated memory or could be applied to weak versus strong memory representations in general. If the latter was the case, then impaired performance of X1-X2 in the delayed group could also be interpreted as the result of a higher interference effect at the time of retrieval due to stronger X1-X3 associations boosted by TMR. However, the fact that the electrophysiological activity differed between groups during TMR suggests that, in our study, an additional neuronal mechanism is being implemented during sleep reactivation in the delayed group. The presence of beta frequencies in the delayed group suggests it is unlikely that the changes in performance are forged only during the retrieval test, lending support to the idea that the weakening of $\mathrm{X} 1-\mathrm{X} 2$ in the delayed group is, at least, initiated during sleep reactivation.

Finally, our experimental approach cannot disentangle the effects of the non-sleep-dependent consolidation process that may have already taken place for X1-X2 memories in the delay group given that they were encoded earlier in the day compared with X1-X2 memories encoded in the contiguous group. Testing for this possibility in future investigation would be important to tighten the role of associative memory strength as a neural property to explain the effects of sleep memory reactivation on complex memory networks.

The current results support the existence of a link between memory reactivation and memory consolidation. The idea that memory reactivation is central in memory consolidation during NREM sleep has received extensive support at the mechanistic level from electrophysiological findings demonstrating rhythmic thalamocortical activity at $12-15 \mathrm{~Hz}$ (termed "spindles"; Contreras et al., 1996), which is coupled to patterns of fast oscillations in the hippocampus $(\sim 200 \mathrm{~Hz})$. This activity is associated with memory replay (termed "ripples"; Wilson and McNaughton, 1994; Buzsáki, 1998; Siapas and Wilson, 1998; Wierzynski et al., 2009), and such patterns of ripple-spindle events are regulated by SOs (0.1-1 Hz; Staresina et al., 2015) which originate in the neocortex (Sirota et al., 2003). Importantly, learning-related variations in spindles and SO properties have been observed in noninvasive electroencephalographic recordings in humans (Gais et al., 2002; Schabus et al., 2004; Clemens et al., 2005; Fogel and Smith, 2006; Marshall et al., 2006). They have been shown to be sensitive measures of the impaired ability to consolidate novel memories through their reactivation during sleep in patients with selective hippocampal damage (Fuentemilla et al., 2013). Our findings indicate that sound cues associated with previously encoded events induce changes in SO, when compared with control sounds presented in an interleaved fashion during target reexposure. However, these SO patterns of change were similar in the contiguous and delayed groups. Thus, if the SO pattern of activity is indicative of memory reactivation during sleep, the fact that target sound cues induced similar changes in SO in both groups suggests that a successful but similar memory reactivation took place during sleep. This raises the interesting question as to whether other neural mechanisms upon successful memory reactivation may in fact account for how overlapping memories are either strengthened or weakened during sleep. In line with this reasoning, we found a concomitant, albeit dissociated contribution of neural oscillatory responses in the theta and beta bands to the contiguous and delayed group. Thus, although theta activity elicited by target cues was similar in the two groups of participants, neural responses in the beta range were only elicited by target sounds in the delayed groups. Therefore, the fact that the same stimulation protocol during sleep elicited distinct neural oscillatory responses in each experimental group provides important insights about the differential nature of the mechanisms involved during memory reactivation. In the context of the current experiment, we speculate that the emergence of neural oscillatory activity in the beta band may be a correlate of the recruitment of additional regulatory brain mechanisms necessary to resolve memory competition occurring between memories reactivated in the delay group. Indeed, previous EEG findings revealed a consistent increase in the beta band $(15-30 \mathrm{~Hz})$ upon memory competition during awake periods which was predictive of the degree of memory forgetting to competing memories during retrieval stages (Hanslmayr et al., 2012). Because of trial number limitations in the current experiment, it was not possible to assess whether such beta response also predicted remembered as opposed to forgotten trials at the within-subject level. Therefore, further studies are required to tighten the link between beta band activity and memory reactivation during sleep.

Forgetting seems disadvantageous but plays an essential role in maintaining the efficiency of memory operations (Anderson, 2003). Previous studies have examined the impact of controlled retrieval on forgetting, whereby executive control processes inhibit or suppress undesirable memories competing for retrieval (Benoit and Anderson, 2012). However, we show that forgetting can operate at the expense of executive control but as an adaptive and unsupervised mechanism triggered during sleep memory reactivation. Our results are in line with earlier (Crick and Mitchison, 1983) and recently revisited (Poe, 2017) frameworks emphasizing the role of sleep in memory weakening of noisy information. The current study provides the first behavioral evidence of memory loss, potentially due to the reactivation of weak associated memories during sleep. This might count as an efficient strategy to prevent the consolidation and integration of unreliable memories that may likely be related to irrelevant events previously acquired during awake time (Kim et al., 2014; Poe, 2017).

In addition, this idea would help bridge the gap between the active systems consolidation view (Diekelmann and Born, 2010) and the synaptic homeostasis hypothesis (SHY; Tononi and Cirelli, 2006). The former explains sleep driven memory benefit as a result of selective synaptic strengthening of memories by the "reactivation" or response of neural activity patterns of the corresponding newly encoded experience. In contrast, the SHY explains sleep memory benefits as a result of a general synaptic downscaling that nullifies the weight of weakly potentiated synapses and ensures the survival of only the "fittest" circuits (i.e., strong circuits). The extent to which the effects observed here relate to one or both mechanisms requires further investigation. Future studies may also incorporate longer periods of sleep including long portions of REM sleep, as this sleep stage has been suggested as underlying several neurochemical responses associated with forgetting during sleep (Poe, 2017). The current data as well as previous studies (Antony et al., 2012; Oudiette et al., 2013) tested the TMR effect on memory consolidation during nap sleep periods (of $40 \mathrm{~min}$ in our design and $\sim 90 \mathrm{~min}$ in others). Thus, although this specific experimental approach has been shown to be effective in promoting memory consolidation across different memory systems (Rudoy et al., 2009; Antony et al., 2012; Hu et al., 2015), investigating during such short periods of sleep may be blind to other sleep-dependent neural mechanisms that may be subject to the alternation of NREM and REM stages during night sleep (Diekelmann and Born, 2010). 
The role of sleep in organizing the storage of complex memories has become latent in the field of neuroscience although it has been difficult to address experimentally. Here, we provide evidence of an adaptive neural mechanism that determines how representations of complex memory networks are shaped during memory consolidation. This process would operate upon the simultaneous activation of individual as well as associated memories and would take place unintentionally during multiple brain states. In fact, the idea that the memory reactivation during sleep strengthened or weakened the associated memories as a function of their related strength fits well with the "nonmonotonic plasticity hypothesis" (Newman and Norman, 2010; Detre et al., 2013). This model has been previously supported by evidence from awake tasks. Our investigation points to this mechanism also in the case of sleep memory reactivation. It therefore draws upon the existence of a general mechanistic principle of memory formation accounting for how the brain consolidates complex networks of interrelated memories.

\section{References}

Anderson MC (2003) Rethinking interference theory: executive control and the mechanisms of forgetting. J Mem Lang 49:415-445. CrossRef

Anderson MC, Bjork RA, Bjork EL (1994) Remembering can cause forgetting: retrieval dynamics in long-term memory. J Exp Psychol Learn Mem Cogn 20:1063-1087. CrossRef Medline

Antony JW, Gobel EW, O'Hare JK, Reber PJ, Paller KA (2012) Cued memory reactivation during sleep influences skill learning. Nat Neurosci 15: 1114-1116. CrossRef Medline

Bendor D, Wilson MA (2012) Biasing the content of hippocampal replay during sleep. Nat Neurosci 15:1439-1444. CrossRef Medline

Benoit RG, Anderson MC (2012) Opposing mechanisms support the voluntary forgetting of unwanted memories. Neuron 76:450-460. CrossRef Medline

Bergmann TO, Mölle M, Diedrichs J, Born J, Siebner HR (2012) Sleep spindle-related reactivation of category-specific cortical regions after learning face-scene associations. Neuroimage 59:2733-2742. CrossRef Medline

Bonnet M, Carley D, Carskadon M, Easton P, Guilleminault C, Harper R, Hayes B, Hirshkowitz M, Ktonas P, Keenan S, Pressman M, Roehrs T, Smith J, Walsh J, Weber S, Westbrook P (1992) EEG arousals: scoring rules and examples. Sleep 15:173-184. CrossRef Medline

Buzsáki G (1998) Memory consolidation during sleep: a neurophysiological perspective. J Sleep Res 7:17-23. CrossRef Medline

Buzsáki G (2015) Hippocampal sharp wave-ripple: a cognitive biomarker for episodic memory and planning. Hippocampus 25:10731188. CrossRef Medline

Clemens Z, Fabó D, Halász P (2005) Overnight verbal memory retention correlates with the number of sleep spindles. Neuroscience 132:529-535. CrossRef Medline

Contreras D, Destexhe A, Sejnowski TJ, Steriade M (1996) Control of spatiotemporal coherence of a thalamic oscillation by corticothalamic feedback. Science 274:771-774. CrossRef Medline

Crick F, Mitchison G (1983) The function of dream sleep. Nature 304:111114. CrossRef Medline

Detre GJ, Natarajan A, Gershman SJ, Norman KA (2013) Moderate levels of activation lead to forgetting in the think/no-think paradigm. Neuropsychologia 51:2371-2388. CrossRef Medline

Deuker L, Olligs J, Fell J, Kranz TA, Mormann F, Montag C, Reuter M, Elger CE, Axmacher N (2013) Memory consolidation by replay of stimulusspecific neural activity. J Neurosci 33:19373-19383. CrossRef Medline

Diekelmann S, Born J (2010) The memory function of sleep. Nat Rev Neurosci 11:114-126. CrossRef Medline

Diekelmann S, Büchel C, Born J, Rasch B (2011) Labile or stable: opposing consequences for memory when reactivated during waking and sleep. Nat Neurosci 14:381-386. CrossRef Medline

Düzel E, Neufang M, Heinze HJ (2005) The oscillatory dynamics of recognition memory and its relationship to event-related responses. Cereb Cortex 15:1992-2002. CrossRef Medline

Düzel E, Penny WD, Burgess N (2010) Brain oscillations and memory. Curr Opin Neurobiol 20:143-149. CrossRef Medline
Eichenbaum H (2000) A cortical-hippocampal system for declarative memory. Nat Rev Neurosci 1:41-50. CrossRef Medline

Fogel SM, Smith CT (2006) Learning-dependent changes in sleep spindles and stage 2 sleep. J Sleep Res 15:250-255. CrossRef Medline

Fuentemilla L, Miró J, Ripollés P, Vilà-Balló A, Juncadella M, Castañer S, Salord N, Monasterio C, Falip M, Rodríguez-Fornells A (2013) Hippocampusdependent strengthening of targeted memories via reactivation during sleep in humans. Curr Biol 23:1769-1775. CrossRef Medline

Gais S, Born J (2004) Declarative memory consolidation: mechanisms acting during human sleep. Learn Mem 11:679-685. CrossRef Medline

Gais S, Mölle M, Helms K, Born J (2002) Learning-dependent increases in sleep spindle density. J Neurosci 22:6830-6834. Medline

Haist F, Shimamura AP, Squire LR (1992) On the relationship between recall and recognition memory. J Exp Psychol Learn Mem Cogn 18:691702. CrossRef Medline

Hanslmayr S, Staudigl T, Fellner MC (2012) Oscillatory power decreases and long-term memory: the information via desynchronization hypothesis. Front Hum Neurosci 6:74. Medline

Hardt O, Nader K, Nadel L (2013) Decay happens: the role of active forgetting in memory. Trends Cogn Sci 17:111-120. CrossRef Medline

Hu X, Antony JW, Creery JD, Vargas IM, Bodenhausen GV, Paller KA (2015) Unlearning implicit social biases during sleep. Science 348:1013-1015. CrossRef Medline

Kim G, Lewis-Peacock JA, Norman KA, Turk-Browne NB (2014) Pruning of memories by context-based prediction error. Proc Natl Acad Sci U S A 111:8997-9002. CrossRef Medline

Lewis PA, Durrant SJ (2011) Overlapping memory replay during sleep builds cognitive schemata. Trends Cogn Sci 15:343-351. CrossRef Medline

Lewis-Peacock JA, Norman KA (2014) Competition between items in working memory leads to forgetting. Nat Commun 5:5768. CrossRef Medline

Maquet P (2001) The role of sleep in learning and memory. Science 294: 1048-1052. CrossRef Medline

Marshall L, Helgadóttir H, Mölle M, Born J (2006) Boosting slow oscillations during sleep potentiates memory. Nature 444:610-613. CrossRef Medline

Martin SE, Engleman HM, Kingshott RN, Douglas NJ (1997) Microarousals in patients with sleep apnoea/hypopnoea syndrome. J Sleep Res 6:276280. CrossRef Medline

Mathur R, Douglas NJ (1995) Frequency of EEG arousals from nocturnal sleep in normal subjects. Sleep 18:330-333. CrossRef Medline

Mölle M, Marshall L, Gais S, Born J (2002) Grouping of spindle activity during slow oscillations in human non-rapid eye movement sleep. J Neurosci 22:10941-10947. Medline

Newman EL, Norman KA (2010) Moderate excitation leads to weakening of perceptual representations. Cereb Cortex 20:2760-2770. CrossRef Medline

Ngo HV, Martinetz T, Born J, Mölle M (2013) Auditory closed-loop stimulation of the sleep slow oscillation enhances memory. Neuron 78:545553. CrossRef Medline

Norman KA, Newman E, Detre G, Polyn S (2006) How inhibitory oscillations can train neural networks and punish competitors. Neural Comput 18:1577-1610. CrossRef Medline

Norman KA, Newman EL, Detre G (2007) A neural network model of retrieval-induced forgetting. Psychol Rev 114:887-953. CrossRef Medline

Nyhus E, Curran T (2010) Functional role of gamma and theta oscillations in episodic memory. Neurosci Biobehav Rev 34:1023-1035. CrossRef Medline

Oudiette D, Paller KA (2013) Upgrading the sleeping brain with targeted memory reactivation. Trends Cogn Sci 17:142-149. CrossRef Medline

Oudiette D, Antony JW, Creery JD, Paller KA (2013) The role of memory reactivation during wakefulness and sleep in determining which memories endure. J Neurosci 33:6672-6678. CrossRef Medline

Peigneux P, Laureys S, Fuchs S, Collette F, Perrin F, Reggers J, Phillips C, Degueldre C, Del Fiore G, Aerts J, Luxen A, Maquet P (2004) Are spatial memories strengthened in the human hippocampus during slow wave sleep? Neuron 44:535-545. CrossRef Medline

Peyrache A, Khamassi M, Benchenane K, Wiener SI, Battaglia FP (2009) Replay of rule-learning related neural patterns in the prefrontal cortex during sleep. Nat Neurosci 12:919-926. CrossRef Medline 
Poe GR (2017) Sleep is for forgetting. J Neurosci 37:464-473. CrossRef Medline

Riedner BA, Vyazovskiy VV, Huber R, Massimini M, Esser S, Murphy M, Tononi G (2007) Sleep homeostasis and cortical synchronization: III. A high-density EEG study of sleep slow waves in humans. Sleep 30:16431657. CrossRef Medline

Rothschild G, Eban E, Frank LM (2017) A cortical-hippocampal-cortical loop of information processing during memory consolidation. Nat Neurosci 20:251-259. CrossRef Medline

Rudoy JD, Voss JL, Westerberg CE, Paller KA (2009) Strengthening individual memories by reactivating them during sleep. Science 326:1079. CrossRef Medline

Schabus M, Gruber G, Parapatics S, Sauter C, Klösch G, Anderer P, Klimesch W, Saletu B, Zeitlhofer J (2004) Sleep spindles and their significance for declarative memory consolidation. Sleep 27:1479-1485. CrossRef Medline

Schreiner T, Rasch B (2015) Boosting vocabulary learning by verbal cueing during sleep. Cereb Cortex 25:4169-4179. CrossRef Medline

Schreiner T, Lehmann M, Rasch B (2015) Auditory feedback blocks memory benefits of cueing during sleep. Nat Commun 6:8729. CrossRef Medline

Siapas AG, Wilson MA (1998) Coordinated interactions between hippocampal ripples and cortical spindles during slow-wave sleep. Neuron 21:1123-1128. CrossRef Medline
Sirota A, Csicsvari J, Buhl D, Buzsáki G (2003) Communication between neocortex and hippocampus during sleep in rodents. Proc Natl Acad Sci U S A 100:2065-2069. CrossRef Medline

Staresina BP, Bergmann TO, Bonnefond M, van der Meij R, Jensen O, Deuker L, Elger CE, Axmacher N, Fell J (2015) Hierarchical nesting of slow oscillations, spindles and ripples in the human hippocampus during sleep. Nat Neurosci 18:1679-1686. CrossRef Medline

Stickgold R, Walker MP (2013) Sleep-dependent memory triage: evolving generalization through selective processing. Nat Neurosci 16:139-145. CrossRef Medline

Tononi G, Cirelli C (2006) Sleep function and synaptic homeostasis. Sleep Med Rev 10:49-62. CrossRef Medline

Tort AB, Komorowski RW, Manns JR, Kopell NJ, Eichenbaum H (2009) Theta-gamma coupling increases during the learning of item-context associations. Proc Natl Acad Sci U S A 106:20942-20947. CrossRef Medline

Wagner U, Gais S, Haider H, Verleger R, Born J (2004) Sleep inspires insight. Nature 427:352-355. CrossRef Medline

Wierzynski CM, Lubenov EV, Gu M, Siapas AG (2009) State-dependent spike-timing relationships between hippocampal and prefrontal circuits during sleep. Neuron 61:587-596. CrossRef Medline

Wilson MA, McNaughton BL (1994) Reactivation of hippocampal ensemble memories during sleep. Science 265:676-679. CrossRef Medline 